\section{Visual backward masking as measured by voice reaction time*}

\author{
CHARLES W. ERIKSEN and BARBARA A. ERIKSEN \\ University of Illinois, Champaign/Urbana, Illinois 61820
}

Instead of using percent correct identifications or detections as the dependent variable, latency in voicing the target stimulus was measured in a backward masking paradigm. Reaction time (RT) to target letters was reliably increased when they were simultaneously encircled by a black ring mask of a size found to produce masking using an identification or detection criterion. The masking function in terms of RT was typical in shape, a decreasing function of stimulus onset asynchrony (SOA) over an interval of 150 msec. Since the target remained "on" when the mask appeared, the results are incompatible with an erasure interpretation of masking effects. Analyses of the variances of the RTs supported an interpretation of a progressive decrease in masking effects as SOA increased.

If a black target letter is flashed in a tachistoscope at a duration long enough to permit identification, the ability to identify the letter is impaired if a black ring circumscribing the letter is simultaneously presented. Presenting the black ring as long as $100 \mathrm{msec}$ following termination of the letter will produce decrement in letter recognition. This phenomenon represents one form of backward masking, a set of phenomena that has been intensively investigated in recent years (see Kahneman, 1968).

There have been two main opposing explanations for this type of masking (Eriksen \& Eriksen, 1971). Integration theory views this phenomenon as the result of a lack of fine temporal resolution by the visual perceptual system. Events separated by very brief temporal intervals are integrated by the visual system and perceived as a composite or montage. Thus, although the target letter and black masking ring may be separated by an interval of 50 msec, the perceptual effect is essentially as though they had been presented simultaneously. The impairment in identification or recognition performance is then ascribed to the same variables that underlie basic visual perceptual acuity.

Sperling (1960) and Averbach and Coriell (1961) have advocated an erasure interpretation of this form of masking. They have suggested that if the stimulus is followed by a second stimulation (mask) before sufficient time has lapsed for the stimulus to be processed, the second stimulation erases the first and replaces it in the visual memory storage (icon).

*This investigation was supported by United States Public Health Service Research Grant MH-1206 and United States Public Health Service Research Career Program Award K6-MH-22014.
Experiments on masking typically have employed percent correct detections or identifications as the dependent variable. ${ }^{1}$ However, if the integration account of masking is correct, reaction time or latency in identifying the target stimulus should also be sensitive to masking effects. Sternberg (1967) has presented evidence indicating that increased processing time is required for degraded stimuli. Thus, if masking is attributable to a degradation of the target due to a montage of target and mask resulting from integration, one should expect to find increased reaction times in identification of the target within the masking interval.

In the present experiment, the Ss were asked to voice the target letter as quickly as possible when it appeared. Single letters were presented at relatively long durations (350 $\mathrm{msec}$ ) followed at various stimulus onset asynchronies (SOAs) by a black masking ring that circumscribed the letter. The SOAs varied from simultaneous to $300 \mathrm{msec}$. Since the target remained on when the mask occurred and both remained on for a relatively long period, any impaired performance could not be attributed to an erasure of the target from the icon or brief visual store. Instead, the visual presentation objectively duplicates the essential condition that an integration theory posits as occurring, i.e., the superimposition of target and mask in iconic representation. Thus, in the present reaction time (RT) arrangement, increased time in vocalizing the target letter would be anticipated when the mask occurred before sufficient time had elapsed to process the target.

Besides shedding light upon the integration vs erasure controversy, a finding that RT was a sensitive measure of masking would expand the techniques available for studying these phenomena. Sternberg's work (1969) has most elegantly demonstrated the analytic power of RT approaches to information processing. One important way that RT data can contribute to understanding masking phenomena is in terms of whether masking is an all or none phenomenon or a continuous but gradually decreasing effect as a function of interstimulus interval (ISI). It is theoretically possible that when masking occurs, it is a complete masking, but the decreasing functions obtained with ISI reflect a decreasing proportion of trials on which this masking occurs. Alternatively, one can conceive of masking as a gradually decreasing effect that occurs in some amount on every trial, but the amount of masking or impairment decreases progressively as the ISI between target and mask increases.

The first theory would be commensurate with a psychological moment interpretation of the integration underlying masking (Kristofferson, 1965; Eriksen \& Collins, 1968). From this point of view, masking occurs when target and mask occur during the same psychological moment and are effectively perceived as simultaneous. As the ISI increases, however, the probability that both target and mask will enter the same psychological moment decreases until the ISI is as long as the duration of a psychological moment. At this time the probability becomes zero that the target and mask will occur in the same moment, and this effectively sets the upper limit of the masking range.

An alternative interpretation that is also consistent with an integration account of masking is that masking occurs on every trial at ISIs within the masking range but that its effect becomes progressively less as ISI increases. The time available for processing the target becomes longer as the mask is delayed, allowing the systems responsible for form identification to process the target more completely before there is impairment of the icon by the masking stimulus.

RT data can provide a means for distinguishing between what might be called the psychological moment hypothesis and the diminishing effect hypothesis. The psychological moment hypothesis would predict that the variance of RTs would increase as SOA increases from simultaneity up to the midrange of the masking function and then decrease as the SOA for which no masking is obtained is approached. If we allow $\mathrm{X}$ to be the mean latency required to process and identify a letter when the mask stimulus is not present, and $X+A$ the mean latency 
Table 1

Percent Errors as a Function of SOA and Ring Size

\begin{tabular}{lccccccc}
\hline & \multicolumn{7}{c}{ SOA (Msec) } \\
\cline { 2 - 7 } Ring Size & 0 & 25 & 50 & 100 & 150 & 300 \\
\hline Small & 7 & 9 & 4 & 7 & 7 & 2 & 6 \\
Medium & 6 & 5 & 3 & 2 & 4 & 3 \\
Large & 6 & 5 & 6 & 3 & 3 & 7 \\
\hline
\end{tabular}

required to identify a letter when full masking is present, then all trials run at $S O A=0$ are drawn from the distribution of $X+A$ and all trials run at a SOA beyond the masking range are drawn from the distribution about $X$. Assuming similar distribution functions for $X$ and $X+A$, at SOA values between 0 and the upper range of masking, differing proportions of trials will be drawn from both the $X$ and the $\mathbf{X}+\mathbf{A}$ distributions, leading to an increase in variance at these SOA values. For example, if the midrange of masking is found to occur at an SOA of $50 \mathrm{msec}$, the psychological moment hypothesis would say that under these circumstances, masking occurred on $50 \%$ of the trials represented by the times the mask and stimulus occurred during the same psychological moment and no masking occurred on the remaining $50 \%$ of the trials, where the mask and the stimulus entered different psychological moments. As SOA is increased or decreased from this value, the proportion of trials that are sampled from one distribution of latencies or the other increases or decreases.

The continuously diminishing effect hypothesis, on the other hand, would lead to the expectation that the variances in latencies obtained over the SOAs that yield masking would remain either constant or, if the variances are correlated with the mean, show a continually decreasing magnitude as the SOA beyond which no masking is obtained is approached.

\section{METHOD}

Subjects

The So were three paid student volunteers (two males) and the senior author. All had normal or corrected to normal vision.

\section{Apparatus and Stimuli}

A Scientific Prototype Model GA three-field tachistoscope was used for presentation of the stimuli. The original lamps had been replaced with Sylvania F4T5CWX bulbs. All fields were maintained at a luminance of $6 \mathrm{~mL}$ and were checked frequently with a Spectra Brightness Spot Meter. Onset of the stimulus was initiated by S using a handswitch, which also started a Hunter Klockounter. A microphone located below the S's viewing hood triggered a Scientific
Prototype 761-G voice relay stopping the Klockounter. Voice RT was recorded in milliseconds.

Stimuli were the capital letters A, E, $\mathbf{R}$, and $\mathbf{S}$ from Paratype pressure letters, 18-point Futura Bold style, placed on transparent vinyl cards. These letters were chosen because the initial vocal sound for all of them occurs at the back of the mouth and all are distinctly different from one another. Since Field 2 was a stimulus field and this field reverses the displayed stimulus, inserting the transparent card into the holder backwards produced a correctly oriented stimulus. Three different sizes of ring stimuli were also placed on transparent cards. The largest ring was designed to be separated on all sides from the stimulus letter by approximately $1 / 2$ deg of visual angle. This ring had an inside diameter of .96 in. and a stroke width of 1/32 in. angle from the nearest point of the stimulus, and had an inside diameter of 69 in. and a stroke width of $1 / 32$ in. The smallest ring (mask) had an inside diameter of .19 in. and a stroke width of $3 / 32$ in. These particular stroke widths were chosen so that the three rings would have approximately equal energy. The total area of the 1/2-deg separation ring was $.10 \mathrm{sq}$ in. and that of the 1/3-deg ring, $.07 \mathrm{sq}$ in. The masking ring had a total area of .11 sq in. Fixation was provided by two dots separated by $.39 \mathrm{deg}$ of visual angle. The stimulus letter appeared directly between them. In all card holders, a white vinyl card was placed in back of the transparent cards in order to provide adequate contrast for the displays.

\section{Procedure}

Stimuli were presented in blocks of 16 trials. Within each block, the four stimulus letters were presented an equal number of times in predetermined random orders, with the restriction that no letter appear more than twice in a row. During each block, presentation of one of the letters was followed by presentation of one of the ring stimuli. Six different delay times for the rings were used, resulting in SOAs of $0,25,50,100$, 150 , and $300 \mathrm{msec}$, the same SOA continuing for an entire block of trials.

On each trial, after a ready signal The middle-sized ring was $1 / 3 \mathrm{deg}$ of from $E, S$ got the fixation field in clear focus and pressed the handswitch. The stimulus letter appeared immediately and remained on for $350 \mathrm{msec}$. The ring followed at one of the SOAs, and it also remained on, terminating at the same time as the letter. The fixation field remained on until the presentation of the ring so that two fields were lighted at all times during the stimulus presentation, maintaining constant Iuminance.

The $S$ identified the letter aloud as quickly as possible. Trials on which $S$ blinked or coughed or was unduly delayed in his response for some reason were rerun later in the block. (Such problems as $\mathrm{S}$ inadvertantly licking his lips or taking a deep breath after pressing the switch occurred from time to time.) In Table 1, the percent of trials on which such errors occurred are shown for each of the ring sizes as a function of SOA. As is seen, the error rate is low and evenly distributed over conditions and SOAs. During each session, all six ISIs were run plus an additional block of trials in which a ring stimulus did not occur. Since it was impossible to present all 18 combinations of three rings by six SOAs within a single experimental session of reasonable length, a Latinsquare design for assigning rings to SOAs was used, with each $\mathrm{S}$ beginning at a different point in the sequence. Three experimental sessions completed all combinations. After one or two practice sescions, each $\mathbf{S}$ participated in nine experimental sessions, yielding three blocks of trials for each SOA by ring combination for each $\mathbf{S}$.

Although three different sized ring stimuli were employed, it was anticipated that only the smallest ring would produce masking effects, since in other research in which percent correct was the dependent variable, rings of the size of the middle and largest ring had not been found to yield appreciable masking effects (Eriksen \& Marshall, 1969; Flom, Weymouth, \& Kahneman, 1963). They were included in the present study primarily as a control for the effects of a second stimulation upon RT. RT has been found in certain circumstances to be sensitive to the occurrence of a second signal following the initial signal to respond (see Herman \& Kantowitz, 1970). By employing two larger sized ring stimuli, a control for the possible effect of a second stimulation is obtained and true masking effects are more adequately evaluated.

\section{RESULTS}

In Fig. 1, mean voicing latency in milliseconds is shown as a function of the SOA between onset of the target letter and the ring stimulus for each of 
the three ring sizes. Also shown as a point in the figure is the mean voicing latency when no ring stimulus followed the target letter. As is clearly evident, a typical backward masking function is obtained for the smallest (masking) ring stimulus. Vocal RT is 40-50 msec longer if the target letter and mask have a simultaneous onset, and it is not until the mask is delayed by 150 msec that voicing latency is as short as that obtained when no mask is presented. The temporal range of the effect is comparable to that obtained in other backward masking studies (Eriksen \& Collins, 1965), and the monotonic shape of the function is also similar (Eriksen, Becker, \& Hoffman, 1970).

Although the effect is not nearly as great, both the 1/3- and the 1/2-deg control rings also show an effect upon RT at SOAs of $100 \mathrm{msec}$ or less. Here the effect is nonmonotonic, with maximum interference occurring at an SOA of 25 msec. Since the effect of these two control rings was appreciably less than that for the masking ring and, in addition, was not expected, an analysis of variance (Ss by Ring Size by SOA) was performed on these data. The main effect attributable to $1 / 2-$ as opposed to 1/3-deg ring size did not approach significance $[F(1,15)=.09, p>.20)$, but the other main effects attributable to SOA $[F(5,15)=4.46, p<.05)$ and Ss were significant. None of the interactions approached significance. Simple $t$ tests for correlated means were run between SOA of 0 and $25 \mathrm{msec}$ for both the 1/2- and the 1/3-deg ring, but for neither ring size did the difference reach significance (p > .05).

In order to evaluate the moment hypotheais against the decreasing effect hypotheais, the variance of RT

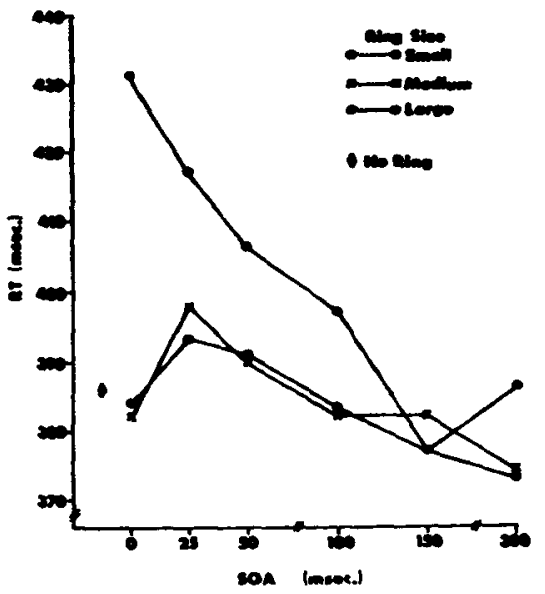

Fig. 1. Mean RTs as a function of SOA for different sized masking rings.

Table 2

Mean Standard Deviations of RTs, Averared Over Ss, for the Small Rin:

\begin{tabular}{cccccccc}
\hline & \multicolumn{5}{c}{ SOA } & (Msec) \\
\cline { 2 - 7 } & 0 & 25 & 50 & 100 & 150 & 300 \\
\hline$\sigma$ & 32.20 & 32.62 & 26.40 & 28.70 & 24.83 & 22.86 \\
\hline
\end{tabular}

at each SOA value from 0 to 300 msec was determined for each $S$ under the masking ring condition. In Table 2 , the mean standard deviation at each SOA value averaged over Ss is shown for the masking or smallest ring condition. As is common with RT data, there is a suggestion that the variances decrease with decreases in mean latency. Most important, however, is the absence of any suggestion that the variances increased to some maximum value in the masking range and then decreased as some psychological moment hypotheses would require.

\section{DISCUSSION}

The results obtained with the masking stimulus bear a striking resemblance to backward masking results obtained in experiments using percent correct as the dependent variable. While it is not logically necessary that the effect of the mask on RT reflect the same underlying mechanism that produces impairment in recognition, nonetheless, such a conclusion seems highly plausible. If we assume that identification accuracy and RT are both reflections of the same underlying "masking" process, then this suggests an interpretation of masking in the context of information processing research.

Sternberg (1967) has found that degraded stimuli require increased processing time, a result that is also in accord with phenomenal experience. An integration account of backwand making is commensurate with this finding, with masking assumed to occur due to degradation of the target stimulus by the mak. Btimulus procesing require time. With brief (10-20 meec) tachistoscopic exposures, it seems clear that recornition or identification of the stimulus is not completed during its phywical duration (Brikeen \& Eriken, 1971). Instead, processing to the point of identification appears to occur from the atimulus trace or, as Neisar (1967) has termed it, the iconic representation. If the mexking stimulus follow before procening of the targat has been completed, the mask in superimposed on the trace of the tanget and processing has to deal with the iconic composite of stimulus and mask.

This interpretation of backward masking suggests new questions, e.g., why does a closely encircling ring degrade a letter and require more time or energy, in terms of exposure duration, for processing to the point of identification? In part, the answer to this question may be in terms of the acuity processes in the visual system, but this essentially begs a question. In all cases, given unlimited exposure, the stimuli involved in these masking studies are such that the eye would be capable of resolving the letter in the ring. But at low energy levels, acuity requirements change. What might be readily resolved at unlimited exposure becomes unidentifiable at brief exposures. This makes clear that the low-energy acuity problem contains a temporal information processing problem.

Minimal energy level in terms either of luminance or exposure duration may be needed for inhibitory processes to maximize contours. However, the results obtained with the 1/3- and the 1/2-deg rings suggest that other components may be involved in masking. As was previously mentioned, these two ring sizes were included in the present experiment primarily as a control for any posible effects of a second stimulus upon RT. It is unlikely that "acuity type" effects are involved here, first because no difference was obtained between these two ring sizes and second because other experimental evidence has sugrested that a separation between contour of a thind of a degree of angle or more does not effect acuity (Flom, Weymouth, \& Kahneman, 1963; Eriksen Rohrbaurh, 1970). Nor wes this separation found to yiold matring effects in a detection study (Brilsen \& Marnhall, 1969). Howevor, muluing ringe separated by this amount from the tariot my not hav been succensful in revenling malking effects due to leas cenditivity using a percent correct dependent variable than is obtained with RT. Or, altarnatively, a different procen may be implicated. It is to be noted that the effect obtained with these lnror ring is greatly reduced relative to a ring of the size typically employed for producing making effects, and that the shape of the function may be different. The larger ring may affect $\mathrm{RT}$ in the present study through some process of attentional diversion or through requiring more complex organizational proceses. The nonmonotonic nature of the function obtained with these larger rings, while not significant, bears 
some resemblance to the U-shaped masking functions discussed by Weisstein (1968), but it would appear quite unlikely that the reciprocal inhibition process posited by Weisstein as responsible for these nonmonotonic functions would be operating with ring stimuli of this size, particularly when only a monotonic function is obtained with the smallest ring.

Examination of the variances of RTs obtained at the SOA values within the masking range gives no support to a psychological moment type of interpretation of integration. Instead, this analysis suggests that the masking process is a gradually decreasing effect with increases in SOA. This would be consistent with the conception of information processing that conceives of various substages preceding the final identification process. The longer the interval between presentation of the stimulus and the appearance of the mask, the further through processing channels the stimulus has proceeded before the mask degrades it. Thus, the mask can have a gradually diminishing effect upon the final processing or the speed with which the processing is carried out.

Finally, it is to be noted that the present results are completely incompatible with the erasure concept of backward masking. In the present experiment, the method of stimulus presentation was such as to preclude the possibility of erasure. The stimulus letters were exposed for a duration of 350 msec, remaining on when the mask was presented. This duration was well within the RT range of three of the Ss. But despite this preclusion of erasure, pronounced effects on RT were found throughout the typical masking range. An erasure concept of masking can remain viable only if the increased RT produced by a masking stimulus in the present experiment is to be attributed to a different process from that responsible for failures in identification or detection that are obtained with briefly presented stimuli.

\section{REFERENCES}

AVERBACH, E., \& CORIELL, A. S. Short-term memory in vision. Bell System Technical Journal, 1961, 40, 309-328.

ERIKSEN, C. W., BECKER, B., \& HOFFMAN, J. E. Safari to masking land: $A$ hunt for the elusive $U$. Perception \& Psychophysics, 1970, 8, 245-250. ERIKSEN, C. W., \& COLLINS, J. F Reinterpretation of one form of backward and forward masking in visual perception. Journal of Experimental perception. Journal of Exp
Psychology, 1965, 70, 343-351.

ERIKSEN, C. W., \& COLLINS, J. F. Sensory traces versus the psychological moment in the temporal organization of form. Journal of Experimental form. Journal of Expe

ERIKSEN, C. W., \& ERIKSEN, B. A. Visual perceptual processing rates and backward and forward masking. Journal of Experimental Psychology, 1971, 89, 306-313.

ERIKSEN, C. W., \& MARSHALL, P. H Failure to replicate a reported U-shaped visual masking function. Psychonomic Science, 1969, 15, 195-196.

ERIKSEN, C. W., \& ROHRBAUGH, J. Visual masking in multielement displays. Journal of Experimental Psychology $1970,83,147-154$.
FEHRER, E., \& RAAB, D. Reaction time to stimuli masked by metacontrast. Journal of Experimental Psychology, 1962, 63, 143-147

FLOM, M. C., WEYMOUTH, F. W., \& KAHNEMAN, D. Visual resolution and contour interaction. Journal of the Optical Society of America, 1963, 53, 1026-1032.

HERMAN, L. M., \& KANTOWITZ, B. H. The psychological refractory period effect: Only half the double-stimulation story? Psychological Bulletin, 1970, 73, 74-88.

KAHNEMAN, D. Method, findings, and theory in studies of visual masking. Psychological Bulletin, 1968, 70, 404-425.

KRISTOFFERSON, A. B. Attention in time discrimination and reaction time. Technical Report 1965 , Contract No. CR-194, National Aeronautics and Space Administration.

NEISSER, U. Cognitive psychology. New York: Appleton-Century-Crofts, 1967.

SPERLING, G. The information available in brief visual presentation. Psychological Monographs, 1960, 74(11, Whole No. 498).

STERNBERG, $S$. Two operations in character recognition: Some evidence from reaction-time measurements. Perception \& Psychophysics, 1967, 2, 45-53.

STERNBERG, S. Memory scanning: Mental processes revealed by reaction-time experiments. American Scientist, 1969 , 57, 421-457.

WEISSTEIN, N. A Rashevsky-Landahl neural net: Simulation of metacontrast. Psychological Review, 1968, 75, 494-521.

\section{NOTE}

1. Fehrer and Raab (1962) employed a $R T$ measure in a metacontrast paradigm and found that RT to the center light was unaffected by the asynchrony of onset of the flanking lights.

(Accepted for publication January 4, 1972.) 